${ }^{1}$ Department of Geological Sciences, University of Michigan Ann Arbor, Michigan, and

${ }^{2}$ Department of Geography, University of Cincinnati, Cincinnati, Ohio, U.S.A.

\title{
The Soil Electric Potential Signature of Summer Drought
}

\author{
S. I. Outcalt ${ }^{1}$ and K. M. Hinkel ${ }^{2}$ \\ With 4 Figures \\ Received October 24,1988 \\ Revised March 20, 1989
}

\begin{abstract}
Summary
During the period from late April to early August, a timeseries of soil electric potential measurements in the upper $15 \mathrm{~cm}$ of mineral soil were collected daily at the University of Michigan Botanical Gardens using an automatic data collection system. These data, after conversion to a surrogate measure of electrolyte concentration, provide a unique record of the 1988 summer drought in a continental location. The effects of rainfall-dewfall electrolyte dilution, evaporation-induced electrolyte concentration and upward-downward soil water advection are well-illustrated in the data. These observations demonstrate that soil electric potential is an easily measured variable of high information content, especially when collected with other system-linked environmental data.
\end{abstract}

\section{Introduction}

The near surface soil layer represents the dynamic transition zone between the atmosphere and the lithosphere. Heat and moisture fluxes are maximized across this layer, and vary temporally in both magnitude and direction (Williams, 1982). Complex chemical reactions occur between atmospheric and biologically-generated gasses, organic compounds, rock forming minerals and soil moisture (Hausenbuiller, 1985).

The soil moisture is an ion-rich solution. Local electrolyte concentration varies with the kinetics of the system and volumetric changes in the solvent due to precipitation, evaporation and internal migration. Changes in the electrolyte concentra- tion over time can be measured directly by obtaining soil moisture samples and determining the concentration of each ionic species present. This methodology provides insights into the types of chemical reactions taking place, but is costly and not conducive to the acquisition of long term, high-frequency data series.

This paper describes a technique used to obtain long-term, high resolution measurements of the electrolyte concentration in the near surface layer. An automatic data collection system was installed to measure electric potential. Four probes were implanted, at various depths, in the upper $15 \mathrm{~cm}$ of a mineral soil at the University of Michigan Botanical Gardens. Recorded once a day, the resulting timeseries covers the period from late April to early August and, after conversion to a surrogate measure of electrolyte concentration, provide a unique and insightful record of the effect of the 1988 summer drought on the soil moisture regime at a continental location. To the best of our knowledge, this is the first time this technique has been used to track changes in the electrolyte concentration of soil moisture as it relates to moisture advection and phase changes.

\section{Instrumentation}

The data collection system was designed for the study of electric potential effects during soil freez- 
ing events, and has been run intermittently since the autumn of 1986. The system is built around an Elexor $1000 \mathrm{H}$, and driven by a Tandy Model 102 laptop computer. The Elexor has $16 \mathrm{~A} / \mathrm{D}$ channels, which are divided equally between thermistor channels and electric potential channels. Probes are connected by cables to the data recording system, which is housed in a small heated building on the experimental plot. The data were written directly to the laptop RAM or to a recording device.

The soil electrode probes were constructed of $8 \mathrm{~mm} \times 5 \mathrm{~cm}$ (outer diameter) copper tubing with three lead wires. Two of the leads are connected to an internal thermistor (Yellow Springs Instruments \#44008), which is embedded in a high thermal conduction sealant within the copper tubing. A third lead is attached to the copper tube for electric potential measurements.

The eight probes, with attached lead wires, were calibrated in an ice slush bath using vendor-supplied data to fit a standard temperature/resistance curve. Temperatures appear to be accurate to within $+/-0.01{ }^{\circ} \mathrm{C}$ over the entire environmental temperatures range, and the system has a $1 \mathrm{mV}$ potential resolution in the range of $+/-4.096 \mathrm{~V}$.

The probes were inserted laterally in two vertical stacks, separated by a horizontal distance of approximately $1 \mathrm{~m}$, at depths of $(0,3,6,9<$ West Site $\rangle) \mathrm{cm}$ and $(0,5,10,15\langle$ East Site $\rangle) \mathrm{cm}$. The probe electrode depths are located relative to the interface of the porous organic debris layer with the underlying pebble-rich sandy loam mineral soil, approximately $1 \mathrm{~cm}$ below the surface. The electric potential is measured between the individual probes (electrodes) and a copper-coated ground spike driven into the soil to a depth of $1.5 \mathrm{~m}$, and located approximately $2 \mathrm{~m}$ from the probe stacks. A more detailed description of the field installation and data acquisition system is available in a recent article (Outcalt et al., 1989).

The system was run at a daily acquisition interval during the early summer of 1988 . The soil electric potential and temperature data were written to the memory of a computer at 00 EST daily, beginning 14 April 1988. On 4 August the system was serviced; probes were extracted for thermal calibration in an ice slush bath and replaced in the soil. The period from the 14th to last (113th) observation of the data set, corresponding to the period from 27 April to 4 August, contained a fascinating record of the early summer drought of 1988. Before this record can be examined, it is necessary to discuss the theory of electric potential in soils and the conversion of electric potential data to a linear surrogate measurement of electrolyte concentration.

\section{Theory}

The equation for the electric potential derived from a concentration cell without transference is given as Eq. (1) below (Prutton and Maron, 1951).

$\varepsilon=-2 \varphi \ln \left(C_{p} / C_{s}\right)$

Here, the potential $(\varepsilon)$ is derived from the electrolyte concentration-activity ratio $\left(C_{p} / C_{s}\right)$ between two electrodes; a soil probe $\{p\}$ and the ground spike $\{s\}$. It is significant that the $\left(C_{p} / C_{s}\right)$ ratio is dependent on the concentration and electrolytic activity of all the ionic species at each probe (Gross, 1967; Yarkin, 1973), and a change in the ratio can be produced by changes both in species composition and concentration. To simplify further discussion, it will be assumed that the ionic species composition at the ground spike and probes is similar, and the ratio $\left(C_{p} / C_{s}\right)$ will be referred to as a concentration ratio. The symbol $(\varphi)$ represents the product of the gas constant and absolute temperature divided by the Faraday constant. Its value at the ice point is approximately $23.5 \mathrm{mV}$.

If $\left(C_{s}\right)$ is taken as the electrolyte concentration at the ground spike and $\left(C_{p}\right)$ as the electrolyte concentration at a near-surface probe, the field situation can be roughly evaluated. Probe potentials are typically in the realm of $300-700 \mathrm{mV}$ relative to the ground spike. Therefore, the soil electrolyte concentration must be much greater at the deep ground spike than at the probes, by a factor ranging from approximately 600 (at $300 \mathrm{mV}$ ) to $2.94 \times 10^{6}$ (at $700 \mathrm{mV}$ ). This finding is consistent with the assumption that the near surface layers are frequently flushed by relatively pure rain and snowmelt waters.

It is also possible to consider the electric potential as a time-dependent change in potential resulting from the present $(C)$ and previous $\left(C^{*}\right)$ electrolyte concentration at a single probe, as shown in Eq. (2).

$\varepsilon=-2 \varphi \ln \left(C / C^{*}\right)$ 
An increase in electrolyte concentration at the probe by a factor of 4 , produced by the evaporation of electrolyte-rich soil water, would produce a potential change of $-65.2 \mathrm{mV}$. In a similar manner, the expulsion of electrolyte from a freezing region or the advection/infiltration of relatively pure water that produces a reduction in concentration by a factor of 4 , would trigger a potential increase of $65.2 \mathrm{mV}$ at an individual probe.

The theory for a concentration cell with transference is derived in a similar manner. The nontransference model was selected because it does not require hydraulic connection between the ground spike and the near-surface probes. The rapid and selective response of the closely nested probes also suggested a non-transference model. The model circuit can be visualized as a potentiometer connected to two jars holding brine, which are themselves connected by a third conductor. The high concentration jar is the analog of the ground spike and the low concentration jar the probe analog. It is implicit that the electrolyte concentration measured by the deep ground spike is not time dependent to a significant degree.

\section{Relative Electrolyte Concentration}

If Eq. (1) is rewritten with the concentration at a soil probe as the objective function, the result is Eq. (3)

$C_{p}=C_{s} \operatorname{Exp}[-\varepsilon /(2 \varphi)]$

As the mid-range of electric potentials is in the realm of $500 \mathrm{mV}$, the ratio of the electrolyte concentration at the ground spike to the probe values is approximately $4.17 \times 10^{4}$. If this value is substituted for $\left(C_{s}\right)$ in Eq. 3, a scale of relative concentrations at the soil probes is produced which is normalized to unity at a potential of $500 \mathrm{mV}$. This scale will be employed in the further analysis and is termed the $C$-index. The $C$-index is a surrogate of electrolyte concentration, on a linear scale. Since the data are more easily discussed in terms of linear relative soil electrolyte concentration rather than non-linear electric potential, the $C$-index facilitates discussion of the timeseries presented in the next section. Due to the extreme drought conditions encountered during the period of record, the $C$-index passed through several orders of magnitude. It was therefore necessary to plot the electric potential timeseries as base 10 logarithms of the $C$-index.

\section{Process Analysis}

Figures 1 and 2 display the plots of $\log 10(C$ index) against the Observation Index (day number of the record) for the two sites. Figure 3 shows the total recorded rainfall during the 24 hour period prior to the observation index of the potential observation. As the rainfall records were collected early in the morning by workers at the Botanical Gardens, the date of late evening and early morning rainfall could be delayed by as much as a day. The rain gauge is located less then $150 \mathrm{~m}$ from the measurement sites.

Figures 1-3 illustrate several interesting characteristics.

(1) Plots of the West Site $(0-9 \mathrm{~cm}$; Fig. 1$)$ and East Site $(0-15 \mathrm{~cm}$; Fig. 2) are nearly identical, and there is little variation in electrolyte concentration at depth.

(2) The $0 \mathrm{~cm}$ electrode, at the organic-mineral soil interface, displays rapid fluctuations at both sites. A comparison of the rainfall record (Fig. 3) and the surface probe fluctuations $(0 \mathrm{~cm})$ shows a dilution of the interface electrolyte by rainwater infiltration.

(3) The rainfall dilution decrease in the $C$-index at $0 \mathrm{~cm}$ is followed by an increase in the $C$-index, which is terminated by a rainfall (or dewfall) event. This increase in concentration is believed to be

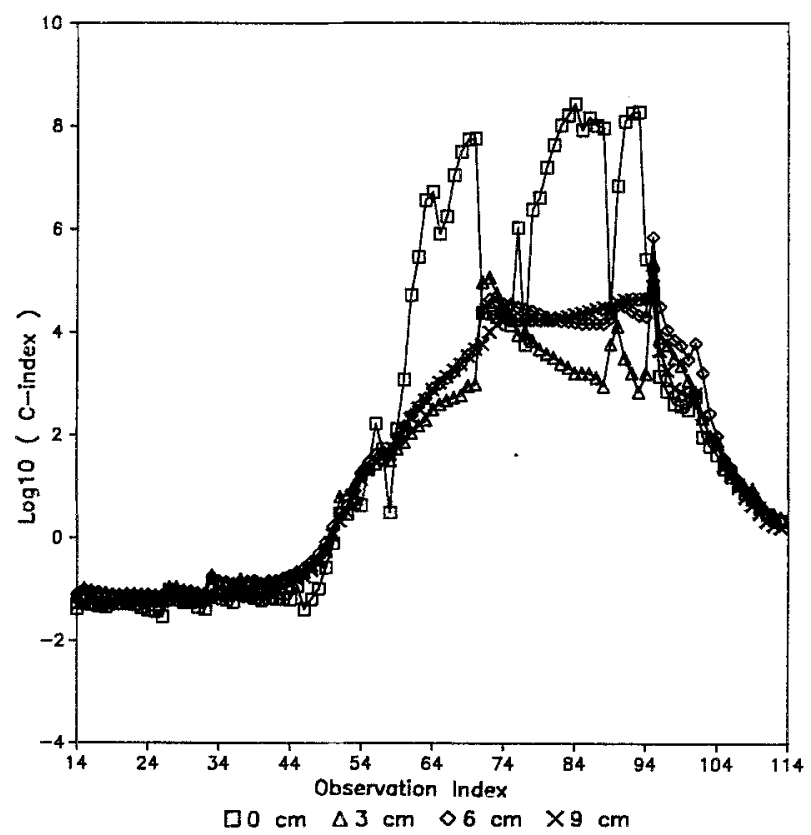

Fig. 1. Electrolyte concentration timeseries at West Site (27 April to 4 August, 1988) 


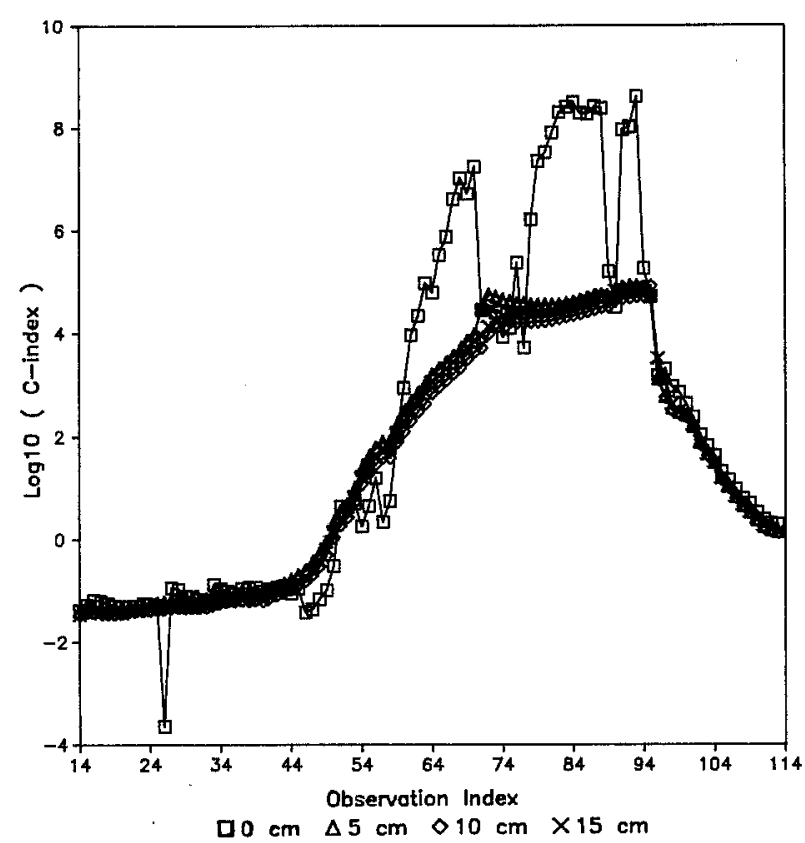

Fig. 2. Electrolyte concentration timeseries at East Site (27 April to 4 August, 1988)

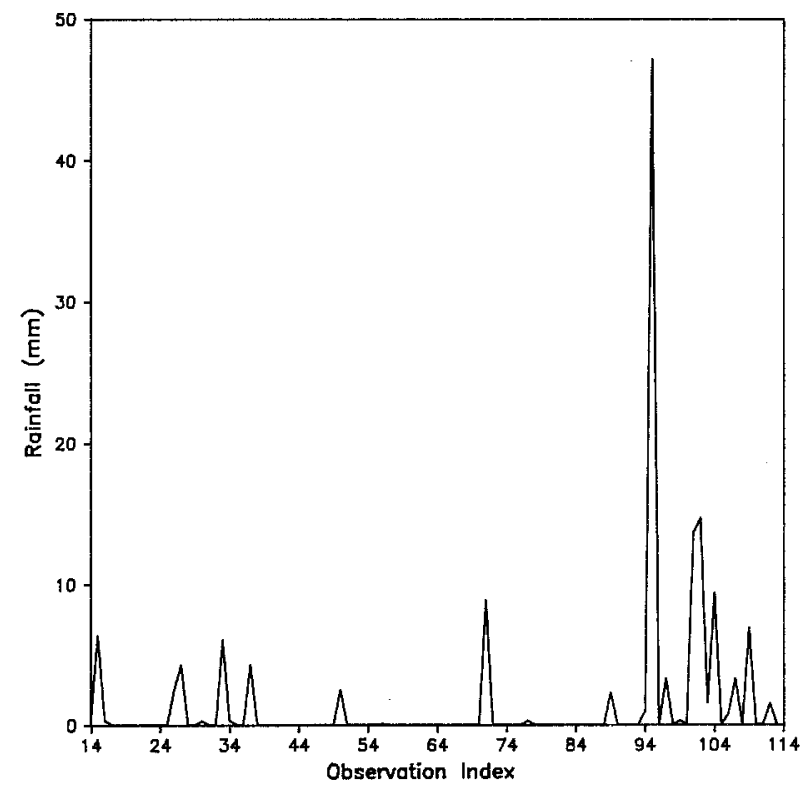

Fig. 3. The rainfall record (27 April to 4 August, 1988)

produced by internal evaporation, which concentrates the electrolyte, and/or by the advection of electrolyte-rich water upward toward the evaporation front.

(4) The decrease of the surface $C$-index at several observation points (for example, at observation indices 46, 56, 64) in Fig. 1 and 2 is not accompanied by a recorded rainfall event, and may be the product of dewfall or unrecorded rainfall.

(5) The concentration amplitude variation produced by rainfall infiltration at observation index 26 at the East Site (Fig. 2) may be the result of site variations in organic mat infiltration capacity.

\section{The Effects of Mid-Drought Rainfall}

In Fig. 4, the $C$-index variations for the West Site probe array and daily recorded rainfall are plotted together for a mid-drought period (16 June to 6 July). Several interesting features of this timeseries are listed below.

(1) The $8.9 \mathrm{~mm}$ rainfall event at observation index 71 was accompanied by a sudden dilution drop in the $C$-index at $0 \mathrm{~cm}$. Concurrently, depth attenuated increases at 3 and $6 \mathrm{~cm}$ may indicate infiltration flushing of electrolyte-rich water from the surface. The gradual increase in the $C$-index at $9 \mathrm{~cm}$ during the first half of the record (66-74) may be the product of upward advection of electrolyte-rich water from depth in response to prolonged evaporation near the surface.

(2) The $0.3 \mathrm{~mm}$ rainfall event at index 77 is accompanied by a rapid drop in the $0 \mathrm{~cm} C$-index only one day after a strong evaporation concen-

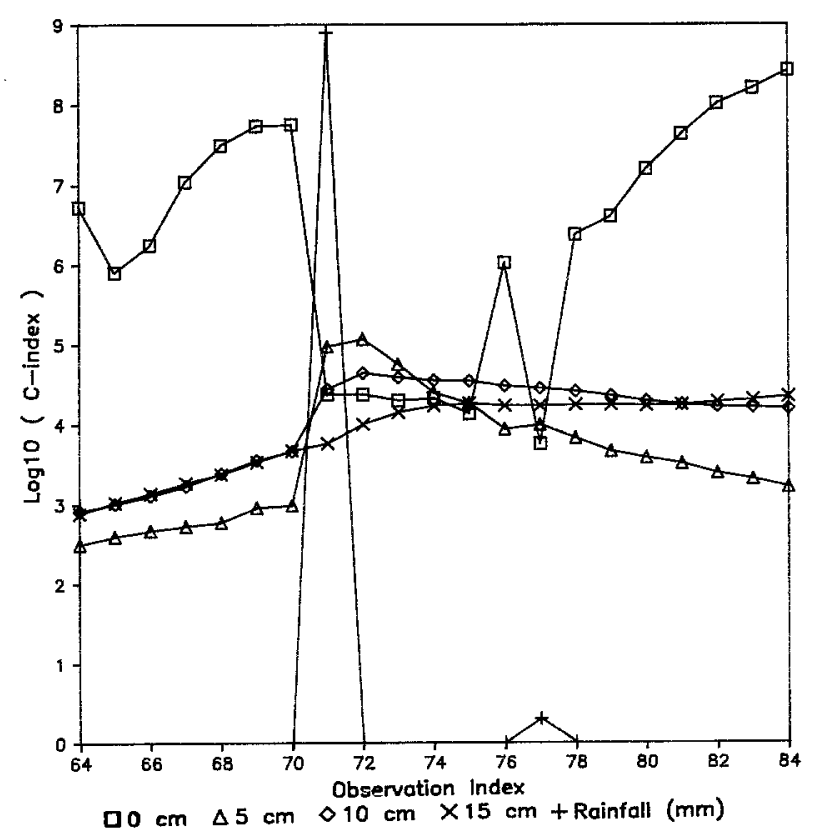

Fig. 4. Mid-drought detail for West Site (16 June to 6 July, 1988) showing electrolyte concentration and precipitation events of 8.9 and $0.3 \mathrm{~mm}$ 
tration upturn. At $3 \mathrm{~cm}$, this event was accompanied by a slight $C$-index increase, presumably resulting from downward infiltration flushing.

\section{The General Pattern}

The general steep upturn of the $C$-index at the deeper probes, commencing near indices 50 ( 2 June 88) leveled-off at a rather high value in the region of index 77 (22 June 88). During this hot, dry period, slight $C$-index fluctuations probably resulted from light rainfall and dew events. Meteorological data obtained from the University of Michigan climate station for this 21 day period shows an average maximum temperature of $29.9 \mathrm{C}$ $(85.9 \mathrm{~F})$ and average minimum temperature of $13.4 \mathrm{C}(56.1 \mathrm{~F})$, with nine of these daytime high temperatures exceeding $32.2 \mathrm{C}(90.0 \mathrm{~F})$. Only $0.5 \mathrm{~mm}$ of rainfall was recorded between 2 June and 22 June at this climate station, which is located approximately $5 \mathrm{~km}$ from the experimental site.

The $47.2 \mathrm{~mm}$ rainfall event occurred in the 24 hour period prior to observation index 95 (17 July 88). This and later rainfall produced a steady decline in the $C$-index at all probes, with minor fluctuations produced by infiltration-evaporation cycling confined primarily to the region of the organic-mineral soil interface.

In short, the ,Drought of $1988^{\circ "}$ at the experimental site reached quasi-steady state conditions during the period from approximately 22 June through 17 July (indices 70-95), during which the effects of mid-drought surface infiltration, resulting from rain and dewfall, failed to refresh the soil below a depth of $10 \mathrm{~cm}$.

\section{Conclusions}

The electrolyte movement during the period of drought is reminiscent of the soil hydrologic regime in more arid regions. Rare rainfall events can saturate the soil to a considerable depth if runoff and evaporative losses are limited. When the evaporation regime is again established, the soluble materials at depth are redeposited in soil pores near the surface. During the next rainfall event, this crust is partially dissolved. The percolating water increases the electrolyte concentration at depth, while near surface soil moisture is diluted by relatively pure rain water.

In southeast Michigan, the climate is suffi- ciently humid that a surface crust is not formed. However, the identical cycle of events occurs in the soil electrolyte concentration. During the recent drought, the water table dropped so dramatically that many wells were closed in local recreational areas, leaving the near surface soil water hydrologically isolated from deeper groundwater. This state is analogous to the steppe lands of the American West where there is no water table at depth. The timeseries $C$-index variations support this view of vertical electrolyte cycling by soil water infiltration and advection to an evaporating front.

The record discussed here indicates that soil electric potential measurements are capable of detecting trace rainfall and dewfall events at the organic-mineral soil interface. The timeseries provide a clear picture of soil electrolyte concentration variations and, by induction, strongly indicate the magnitude of other processes (internal evaporation, infiltration flushing and rainfall-dewfall dilution). Electrodes can be fabricated by stripping insulation from extremely fine wire and, therefore, a much higher spatial resolution is possible than that presented here. Interested readers may wish to add soil electric potential to their current or proposed measurement systems, particularly those which are to be deployed at remote field sites to collect system-linked environmental data (e.g., Parameswaran and Mackay, 1983).

\section{Acknowledgements}

Funds for the construction of the data acquisition system were provided by a development grant from the Office of the Vice-President for Research, Linda S. Wilson, of the University of Michigan. The system could not have been made operational without the extensive labors of Scott Baird, Electronics Technician, Department of Geological Sciences, University of Michigan. The manuscript was prepared by Mrs. Bevelyn Mueller, University of Cincinnati.

\section{References}

Gross, G. W., 1967: Freezing potentials in the system $\mathrm{H}_{2} \mathrm{O}-\mathrm{NH}_{3}-\mathrm{CO}_{2}$ at controlled concentrations. J. Atmos. Sci., 28, 1005-1014.

Hausenbuiller, R. L., 1985: Soil Science: Principles and Practices, Third Edition. Dubuque: Wm. C. Brown, 610 pp.

National Climate Data Center: Climatological data, Michigan, June 1988. Volume 103, Number 6.

Outcalt, S. I., Gray, D. H., Benninghoff, W. S., 1989: Soil temperature and electric potential during diurnal and seasonal freeze-thaw. Cold Regions Sciences and Engineering (in press). 
Parameswaran, V. R., Mackay, J. R., 1983: Field measurements of electrical freezing potentials in permafrost areas. In: Proceedings, Fourth International Conference on Permafrost, 17-22 July 1983, Fairbanks, AK, 962-967.

Prutton, C. F., Maron, S. H., 1951: Fundamental Principles of Physical Chemistry. New York: MacMillian Company, $803 \mathrm{pp}$.

Williams, P. J., 1982: The Surface of the Earth. New York: Longman, $212 \mathrm{pp}$.
Yarkin, I. G., 1973: Effect of natural electric potentials on water migration in freezing soils. In: Permafrost: U.S.S.R. Contribution to the Second International Conference, pp. 359-361.

Authors' addresses: S. I. Outcalt, Department of Geological Sciences, University of Michigan, Ann Arbor, MI 48109-1063 U.S.A. K. M. Hinkel, Department of Geography, University of Cincinnati, Cincinnati, Ohio, OH 45221-0131, U.S.A. 